and planning how to implement it. Engagement of senior managers and the wider MDT were key change facilitators, as was a comprehensive education programme for staff and volunteers. Both research projects identified a number of organisational and operational challenges in terms of: concept, lay/ external perceptions, alignment with current models of care and a tradition of 'over-caring', risk aversion, strategic direction, senior/management engagement, insufficient time and/or resources, variable condition of patients, poor team working/ communication.

Conclusion PAR can be used to integrate rehabilitative palliative care, and HCPs want to engage with and implement the concept in their practice. The combined results of these two research studies show how research can drive a change in practice successfully through a top down and bottom up approach.

\section{0-15 AN EXERCISE AND NUTRITIONAL REHABILITATION (ENERGY) TRIAL IN PATIENTS WITH INCURABLE CANCER}

\begin{abstract}
${ }^{1,2}$ Charlie Hall, ${ }^{1} J a n e$ Cook, ${ }^{1}$ Honor Blackwood, ${ }^{1}$ Erna Haraldsdottir, ${ }^{1}$ Duncan Brown, ${ }^{3}$ Matthew Maddocks, ${ }^{4}$ Liz Dixon, ${ }^{5}$ Richard Skipworth, 1,2 Marie Fallon, 1,2 Barry Laird. ${ }^{1} S t$ Columba's Hospice, Edinburgh, UK; ${ }^{2}$ The University of Edinburgh, Edinburgh, UK; ${ }^{3}$ Cicely Saunders Institute of Palliative Care, Policy and Rehabilitation, London, UK; ${ }^{4}$ Southampton Clinical Trials Unit, Southampton, UK; ${ }^{5}$ Royal Infirmary, Edinburgh, UK
\end{abstract}

\subsection{6/bmjspcare-2019-HUKNC.15}

Background Rehabilitation is an essential component of palliative care (Tiberini \& Richardson, 2015), however, data on efficacy and key components are lacking (Salakari, Surakka, Nurminen et al., 2015). There is a strong argument that any rehabilitation programme should combine exercise and nutrition, which together may improve physical function and quality of life, but this needs careful evaluation (Payne, Larkin, McIlfatrick, et al., 2013; Chasen, Bhargava, MacDonald, 2014). This is being done through the ENeRgy trial which compares an Exercise and Nutrition based Rehabilitation programme with standard care, in patients with incurable cancer. The trial was funded by Marie Curie and the Chief Scientist Office.

Aims The primary aim was to assess the feasibility of an Exercise and Nutrition based Rehabilitation programme. Secondary aims included assessment of quality of life (patient and partner-carers), functional and nutritional status, contamination of the control group, health economic impact and survival.

Methods A single centre, randomised, unblinded feasibility trial was undertaken. Patients were randomised to receive an exercise and nutrition based rehabilitation programme (intervention) or standard care (control). Eligible patients were: $\geq 18$ years, Karnofsky Performance Status $\geq 60$, with a diagnosis of incurable cancer; not undergoing anti-cancer treatment.

Results 45 patients were recruited from a mixture of hospices and a cancer centre. Challenges included optimising patient recruitment, compliance with the components of the rehabilitation programme, and the establishment of a clinical research team within an independent hospice. Patients' overall experience of the rehabilitation trial was positive and nurtured a positive, empowering impression of the hospice setting. Key themes and some preliminary data will be presented.

Conclusions The ENeRgy trial is an important step in assessing the feasibility of an outpatient, hospice based rehabilitation programme for patients with incurable cancer and will inform future work in this area.

\section{$0-16$ SUPPORTING FAMILY CARERS OF PEOPLE WITH MOTOR NEURONE DISEASE: ADAPTATION OF THE CSNAT INTERVENTION}

${ }^{1}$ Gail Ewing, ${ }^{2}$ Sarah Croke, ${ }^{2}$ Christine Rowland, ${ }^{2}$ Gunn Grande, ${ }^{2}$ Alex Hall. ${ }^{1}$ University of Cambridge, Cambridge, UK; ${ }^{2}$ University of Manchester, Manchester, UK

\subsection{6/bmjspcare-2019-HUKNC.16}

Background Family carers play a vital role in supporting people with Motor Neurone Disease (MND) but face substantial physical and mental demands in doing so. Timely support from healthcare professionals is crucial to enable carers to fulfil their caregiving role and to look after their own wellbeing. The Carer Support Needs Assessment Tool (CSNAT) intervention is an evidence-based approach for person-centred assessment and support. This project aimed to: (i) adapt the CSNAT intervention to the context of MND care; (ii) test implementation of the adapted CSNAT-MND intervention within specialist MND services.

Methods Stage 1: focus groups and interviews with 14 bereaved and 19 current carers to explore support needs (met and unmet) and supportive inputs at key stages of the patient's illness. Stage 2: workshops with 24 practitioners and 19 carer advisers to adapt the content of CSNAT intervention and its delivery within MND care. Stage 3: implementation of CSNAT-MND in three specialist MND services; interviews with practitioners and carers exploring their experiences of the intervention.

Results Content of CSNAT-MND included an additional domain on support with relationships. Intervention implementation adapted to (1) legitimise assessment of carer support needs as part of routine care in MND by practitioners, (2) delivery of a stand-alone introduction stage of the CSNATMND intervention and (3) provide a dedicated assessment and planning conversation at a subsequent contact. This basic model was then suited to fit the working practices of three different MND services. We will present challenges of tailoring and implementing the CSNAT-MND intervention in real life practice.

Conclusion Implementation of the CSNAT-MND requires the establishment of a clear protocol defined at service level. This project provides insights into how carers of people with MND want to be assessed and supported, how this process can be delivered, and the feasibility of implementing the CSNAT-MND in practice.

\section{0-17 LEGAL NEEDS TOWARDS END OF LIFE: DEFINITION EXPERIENCE AND IMPLICATIONS FOR POLICY AND PRACTICE}

${ }^{1}$ Colette Hawkins, ${ }^{2}$ Helen Close, ${ }^{3}$ Margaret Kirby, ${ }^{4}$ Hannah Hesselgreaves, ${ }^{2}$ Charlotte Rothwell, ${ }^{5}$ Sarah Beardon, ${ }^{5}$ Hazel Genn. ${ }^{1}$ St Oswald's Hospice, Newcastle, UKi ${ }^{2}$ Newcastle University, Newcastle, UK; ${ }^{3}$ Legacare, Newcastle, UK; ${ }^{4}$ Northumbria University, Newcastle, UK; ${ }^{5}$ University College, London, UK

\subsection{6/bmjspcare-2019-HUKNC.17}

Background Life-limiting illness generates an array of challenges for patients and carers but little is known about legal issues. There is no definition of legal issues in this context and limited integration of care across health, social, advice, legal and charitable sectors although all contribute to holistic care. 
Aims The Legal Needs of Adults towards End of Life (LeNA) project investigated the scope and scale of legal needs from a range of perspectives, the support structures available and professional education needs with a view to shaping national policy.

Methods Sept 2017 - June 2018: systematic literature review and national stakeholder engagement exercise across health, social, charitable, advice and legal services: definition of legal needs, available support and gaps. May 2018 - March 2019: semi-structured interviews (24 patient/carer) and questionnaires (124 patient/carer/staff) to evaluate experience of legal needs. Sept 2018 - Jan 2019: four inter-professional workshops to consider educational needs through a taster session and focus groups (6).

Results Legal needs are very broad and practice highly variable. The project has developed a classification relating to patient/carer rights and professional responsibilities. This could direct future policy. Patients and carers struggle with unmet needs, uncertain of how to access help and unwilling to plan for the future in the context of active concerns. Some support structures already exist but the project identified a number of barriers and consideration needs to be given to accessibility and suitability of these. There is significant enthusiasm for widely inter-professional education in this area, using an interactive group structure.

Conclusions Legal needs are poorly defined in palliative care, resulting in variability in practice and unmet need. This is an area for improvement and follow-on research is planned at national policy level. Together with novel education, this will support better assessment and integrated care around legal needs towards end of life.

\section{Parallel Session 5: Workforce Support and Development}

\section{0-18 WELCOMING FREDIE}

Dallas Pounds. Royal Trinity Hospice, London, UK

10.1136/bmjspcare-2019-HUKNC.18

Royal Trinity's mission includes 'challenging and overcoming barriers to equality, diversity and inclusion'. We want to be an inclusive and fair workplace and service provider where potential can be fulfilled and care delivered, unencumbered by real or perceived barriers relating to protected characteristics, and our uniqueness as individuals.

Our track-record of championing LGBT + needs in our staff and our patients is well known, but we wanted to go further. 'Welcoming FREDIE' benefits our entire workforce by broadening our focus to all protected characteristics and adopting 'FREDIE' (fairness, respect, equality, diversity, inclusion, engagement) as part of the Trinity family, as part of our culture.

Starting in 2018, with our sights set on achieving an Investors in Diversity level 2 accreditation, and with an eye to our staff survey results, our aim was to develop an even happier, more committed workforce that was more comfortable to bring the best version of themselves to work every day, and were more aware of legally protected characteristics in their patients and colleagues and more aware of their own unconscious biases.

Actions have included:

- a 20 week programme of internal events and education 'FREDIE Fortnights', dedicating a fortnight to each protected characteristic with another dedicated to mental health;

- changes to Trinity's mandatory e-learning, manager training, and appraisal process

- updating policy and strategy documents;

- contracting an external helpline for our workforce to report harassment and discrimination.

Impact so far:

- In January 2019, Trinity was named number 84 in the National Centre for Diversity's Investors in Diversity Top 100 Index, the first hospice to be listed in the Index and one of only two healthcare providers nationally;

- In June 2019, Trinity was shortlisted for a Nursing Times Workforce Award for 'Welcoming FREDIE', and nominated for a National Diversity Award as a community organisation;

- Improved staff satisfaction and awareness.

\section{0-19 STAFF WELLBEING IN UK CHILDREN'S HOSPICES (SWICH): A MIXED-METHODS STUDY}

${ }^{1}$ Andrew Papworth, ${ }^{2}$ Jan Aldridge, 'Bryony Beresford, 'Lorna Fraser, 'S Suzanne Mukherjee, 'Johanna Taylor. 'University of York, York, UK; ${ }^{2}$ Martin House Hospice, Wetherby, UK

10.1136/bmjspcare-2019-HUKNC.19

In the UK, the children's hospice sector has become increasingly concerned about levels of work-related stress among its staff. This stems from increasing evidence that staff wellbeing is associated with the quality, cost and safety of patient care, and also an acknowledgment of the important role of those working in children's hospices. It is therefore important to review and develop systems to enhance staff wellbeing and reduce the risk of occupational burnout.

The SWiCH study, conducted by the Martin House Research Centre at the University of York, is a national survey of staff wellbeing in UK children's hospices. It aims to increase understanding about the work-related stressors and rewards experienced by children's hospice care staff, and to identify the staff support systems and organisational practices that offer the most potential to enhance wellbeing at work.

This paper will present findings from the first phase of this ongoing study. This consists of two elements. The first is a systematic review aimed at identifying the factors associated with the psychological work-related wellbeing of hospice staff, and reviewing evidence on levels of burnout amongst hospice staff. Literature searches have identified 86 international studies that have explored this in hospice settings. The second element is the development and validation of a new tool to measure work-related stressors and rewards in children's hospice staff. This involves adapting existing scales developed for use in paediatric oncology through surveys, focus groups and interviews with staff in six children's hospices.

Findings from this first phase will inform the development of a national survey of all UK children's hospice organisations and their care staff. 